\title{
Design of Mobile Gateway for Implementation of Smart Work System
}

\author{
Sang-Young $\mathrm{Oh}^{1}$ and Bong-Hyun Kim ${ }^{2}$ \\ ${ }^{1}$ Department of Business Administration, Youngdong University, 310 Daehak-ro, Youngdong-eup, Youngdong-gun, \\ Chungcheongbuk-do 370-701, Republic of Korea \\ ${ }^{2}$ Department of Computer Engineering, Kyungnam University, 7 Kyungnamdaehak-ro, Masanhappo-gu, \\ Changwon-si, Gyeongsangnam-do 631-701, Republic of Korea
}

Correspondence should be addressed to Bong-Hyun Kim; hyun1004@uok.ac.kr

Received 28 August 2014; Accepted 8 October 2014

Academic Editor: James J. Park

Copyright (C) 2015 S.-Y. Oh and B.-H. Kim. This is an open access article distributed under the Creative Commons Attribution License, which permits unrestricted use, distribution, and reproduction in any medium, provided the original work is properly cited.

In the development of new technologies based on mobile system, there is a growing interest as a fundamental technology. In particular, in order to realize a mobile office and mobile phones, conferencing remote document is a technique that can realize smart network services solutions with enhanced real-time communication, real-time information sharing, and collaboration. Therefore, in this paper, the design meets the diverse needs of customers and the smart work platform mobile-based fast and conveniently it is trying to develop. For this reason, I have developed a mobile gateway that is based on the communication server construction OPEN API development, management of mobile ID, protocol design, and design of SSL/TLS security tunnel. Also, we developed a smart work platform that you apply this, you are trying to provide information systems environment of mobile company.

\section{Introduction}

In the sense of recent office moves, mobile office is increasingly popular. In other words, the smart phone is opening the era of the popularization of the mobile Internet, starting your day with a smartphone, finishing with a smart phone; the influence of mobile generation is increasing [1]. However, there is a drawback operating system for mobile devices and the kind of smartphone that limited Korea. In addition, the intended uses are also limited to personal use; the introduction of business-based solution that can be utilized in educational institutions and companies has been accelerated. Smartphone is more likely to be used as a personal office equipment as well as necessary office equipment and supplies used by personal PC, a notebook in business there is a need for this type of software development work. Reflecting this, the office is used to mean a place where smart phones. In other words, the introduction of mobile office companies to increase steeply advantages of immediate, short time, productivity, efficiency, increase usability, competitive $[2,3]$.
Therefore, in this paper, I try to develop a smart work platform of mobile gateway-based rapid and inexpensively to meet the diverse needs of our customers (Figure 4). For this reason, the development of mobile gateway can be used as a first stage, to be shared by various applications and Web services identity information of the user. In two stages, the gateway between the mobile terminal and the SSL/TLS End-toEnd strengthen the security services provided through a security tunnel. In order to provide office services from the terminal finally subject was studied by developing smart work platform.

\section{Current Situation of Technique}

2.1. Domestic Technology Trend. Domestic mobile office market was a time when the extent of setting up a wireless network in your company has been to represent mobile office 10 years ago. The main terminal of the first generation of mobile office environment had a wireless network device is utilized notebook PC. It came into the second generation that the 


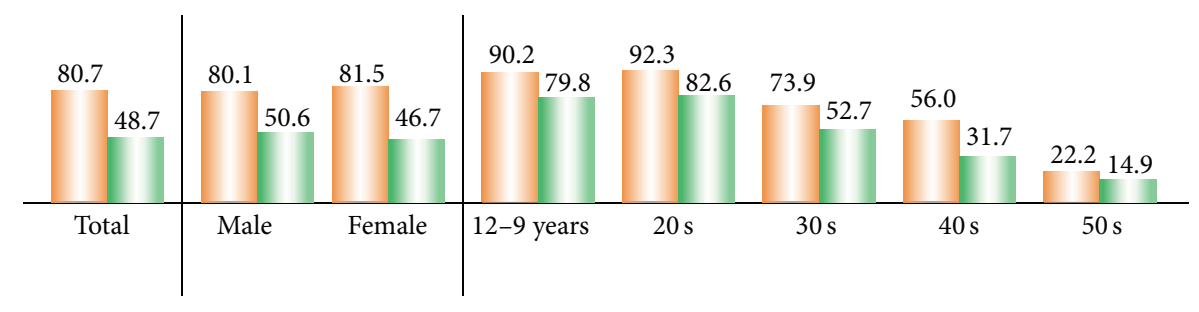

Smartphone
Cellular

Figure 1: Wireless Internet usage.

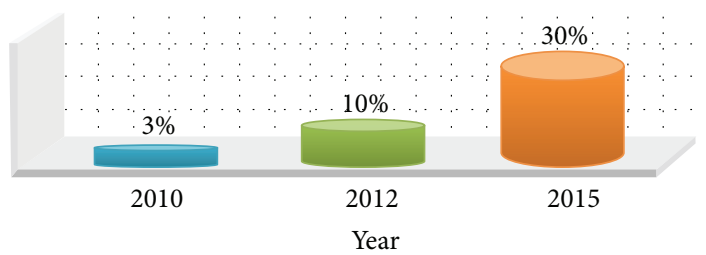

FIGURE 2: Smart work creates an environment of workers of 30\% in 2015.

mobile device is more diverse, will be started to utilize a variety of mobile devices including PDA, smart phone, tablet PC (Figure 3). Currently, many companies are utilizing the mobile office on the level of the second generation. Service can include intranets within the corporate from the mobile device and is used in conjunction with customer relationship management (CRM), enterprise resource planning (ERP), Supply Chain Management (SCM), and so forth, Step 3 mobile office. It is possible to retrieve service related information, such as when this step is that the user has a smart phone or a PDA, a tablet PC. Another object of the present invention is a mobile office environment which can be processed in real time to conduct a wide range of activities such as a remote document approval that is mounted. Currently, technology solutions in domestic is a state that depends on overseas market solutions in the entry time. Domestic is the rudimentary stage which entered the market formed, but mobile office market is expected to grow to 5.9 trillion yen in 2014 from 2.9 trillion won in $2009[4,5]$.

2.2. Overseas Technology Trends. If going overseas is a step of fixing the service to starting with the IBM a mobile office service performed by a program called Smart (SMART, Space Management And Required Technology). It looks like it will continue to grow to $\$ 90$ billion in 2015 from $\$ 61$ billion in 2009 (Figure 2).

British Telecom (BT) was the first that started in Europe FMC service, but due to technical limitations, targeted at individual customers "BT Convergence" service to give up, to concentrate on the $\mathrm{B} 2 \mathrm{~B}$ market that they now have. On the other hand, they issued a "Wi-Fi Calling for Business" based on the UMA in conjunction with Blackberry; T-mobile is a solution that extends the mobile communication PBX such as Avaya and Cisco in (Private Branch eXchange) systems provide to AT\&T. British Telecom (BT) may be able to connect the Internet, mobile phone, and wireless Internet access via a (MobileXpress SW) Mobile Express software, to perform a business process by connecting to the application or the company intranet from anywhere in the world possible (Figure 1) [6, 7].

Now, I support up to 650 pounds cost of Internet connection, such as the purchase of e-mail forwarding service (P.O. Box) and of office furniture in the telecommuter. As a result, productivity is increased to $20-60 \%$ from the residence office staff, sick leave absence rate of employees was reduced $63 \%[8]$.

\section{Implementation of Smart Work System}

In this paper, we conducted a study to develop a smart work platform of mobile gateway-based convenient and quick to fit different requirements of our customers (Figure 5). For this reason, depending on the smart work platform is in conjunction with the company's existing information systems and we have developed a smart work platform of mobile gateway base for small- and medium-sized enterprises, which can significantly reduce building operating costs. Finally, we conducted a survey by classifying the development of smart work platform to which the development of the mobile gateway is applied $[9,10]$.

The mobile gateway was developed by classifying into three stages. In one step, we have developed an API in conjunction with the integrated management of mobile ID. Therefore, it is managing the identities to be used and offline services have been developed in the form of the Open API. We also develop a management protocol issue of mobile ID, update, disposal, user-friendly and establish trust early we have developed a key technology exchange and efficient 

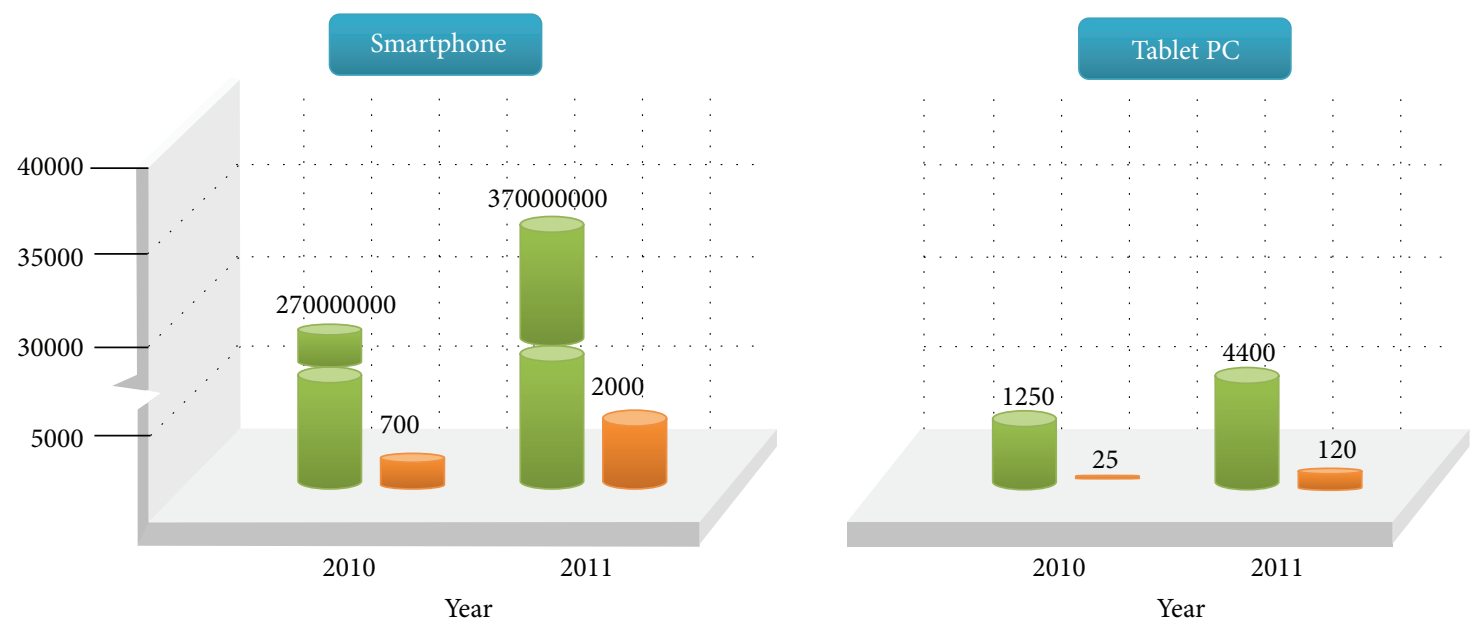

(Unit: ten thousand)

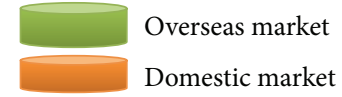

Figure 3: Smart device usage.

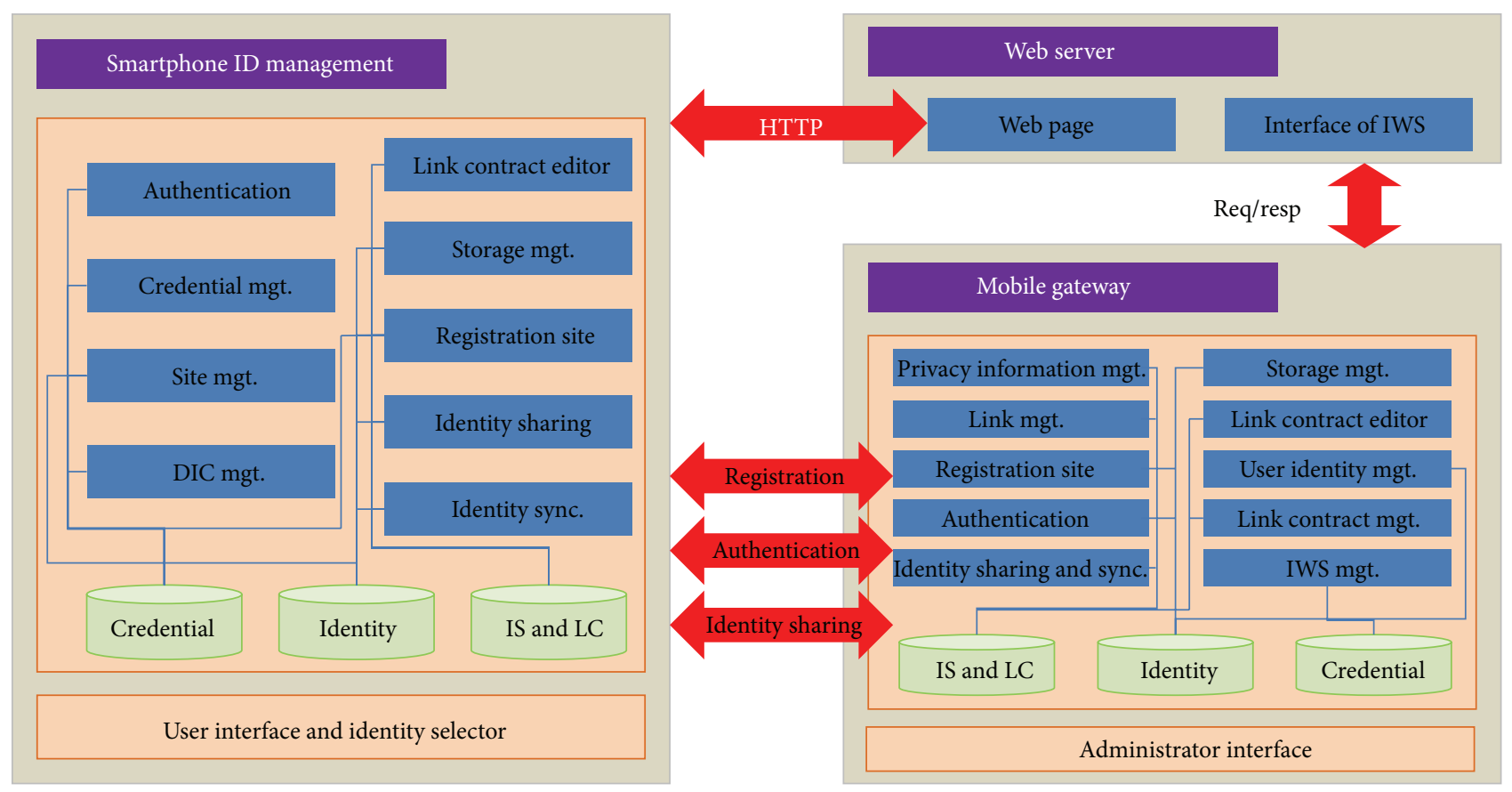

FIgURE 4: Design of mobile gateway.

authentication. In Step 2, we have developed the security features of End-to-End and terminals. Therefore, we have developed a SSL/TLS tunneling security to provide security features of End-to-End and terminals. In step 3, was developed for embedded mobile gateway. Therefore, we developed a mobile gateway box using embedded OS, which was communicating with the mobile office server for PC. Also, I have to prepare a gateway that takes into account the performance and scalability [11].
In the smart work platform, based on the mobile gateway, we have developed a server/client form. Smart work platform of mobile gateway-based has developed a remote management capabilities authentication, LBS/GIS, of the terminal. In other words, I have developed a smart work platform that provides services such as version control of remote administration of client terminal, authentication, LBS/GIS, and the mobile office. In addition, we have developed a data conversion function of the cellular phone. As a result, it 


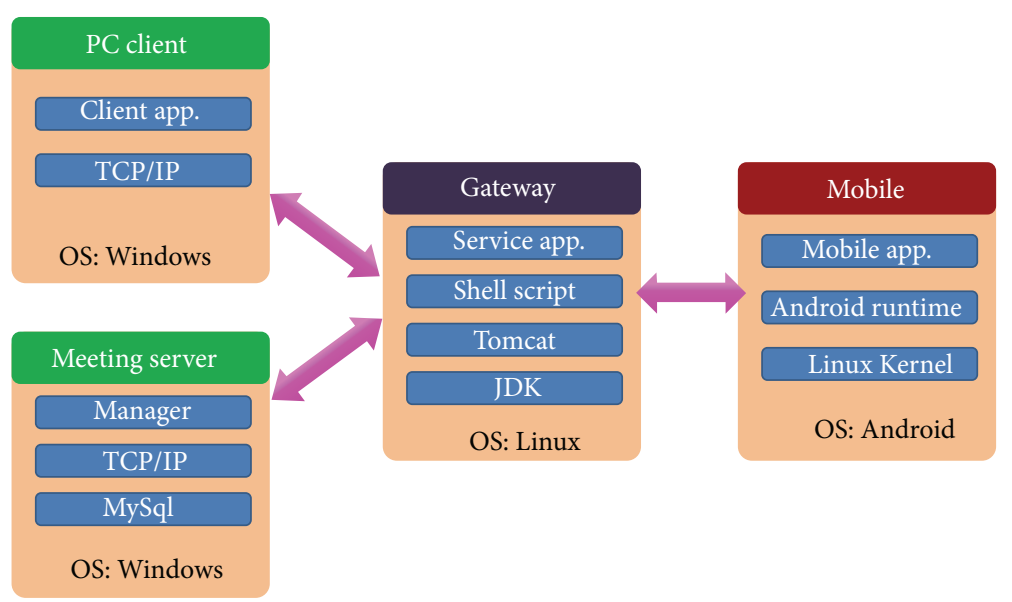

FIGURE 5: Smart work system construction plan.

provides a mobile data conversion function that specializes in business process MS Office document, HWP, such as PDF. Finally, I have developed a smart work platform based on server/client architecture [12].

Smart work platform server is provided to register and manage the terminal, conference registration and management of data, the mobile client deployment, and update capabilities. Then, in the client platform of smart work, I provide smartphone, mobile services and agent platform, and a mobile services platform electronic program.

In the field of mobile gateway-based server platform technology, smart work platform of mobile gateway-based, which was developed in this paper, it is possible to expand the mobile Web server application art. Further, it is possible by technical developments for executing Web server from the mobile terminal, to develop SW which operatively interconnects the PC environment with the mobile module. As a result, it is possible to develop the new technology of the next generation, such as remote monitoring collaboration support system and real-time decision making of mobile phone-based, real time.

\section{Conclusions}

In this paper, we studied a technique capable of releasing a convenient smart work business mobile market is steadily increasing current is applied. Therefore, we have developed a smart work platform mobile gateway base capable of increasing the efficiency of a company's information system. Smart work platform was developed integrated management of mobile ID in the form of Open API, identity management, protocol management and developed a mobile gateway to the application of the security technology of End-to-End.

In addition, the smart work platform, authentication, remote management of the terminal, data conversion, and developed server/client technology were applied.

\section{Conflict of Interests}

The authors declare that there is no conflict of interests regarding the publication of this paper.

\section{References}

[1] H. W. Jeong, "Smartphones and future changes," Journal of Korean Contents Society, vol. 8, no. 2, pp. 28-33, 2010.

[2] H. Amer, Smart Work U: Get Your Degree the Smart Way-Save Time and Money, LifeNotes Press, 2011.

[3] S. Y. Yu and K. S. Noh, "The power analysis of smart work industry and increase plan," Journal of Korean Digital Policy \& Management Society, vol. 9, no. 6, pp. 187-196, 2011.

[4] Impact Press, Condition and Prospect of Smart Work \& Smart Office, Impact Publications, 2012.

[5] J. G. Ryu, A study on smart work promoting Korean policy [M.S. thesis], Dankook University, Yongin, Republic of Korea, 2012.

[6] B. Y. Oh, A study on business process reengineering and information model of smart office for public agencies [M.S. thesis], Korea Univ. A Master's Degree Paper, 2011.

[7] H. B. Ko, H. D. Lim, and J. I. Lee, "Policy directions and strategies for boosting cloud computing-based smart mobile office," Journal of Korean Information Science Society, vol. 259, pp. 13-19, 2010.

[8] Daco Industry Laboratory, Condition and Strategies of Smart Work Mobile Office, Daco Industry Laboratory, 2011.

[9] D. G. Lee and J. H. Lim, "An implementation of mobile gateway based on android smartphone," Journal of Korean Digital Policy \& Management Society, vol. 12, no. 1, pp. 333-338, 2014.

[10] H.-Y. Hsueh, C.-N. Chen, and K.-F. Huang, "Generating metadata from web documents: a systematic approach," HumanCentric Computing and Information Sciences, vol. 3, article 7, 2013.

[11] H. J. Seo and Y. C. Choy, "ID credit scoring system based on application scoring system: conceptual online ID credit for ID integrated environment," Journal of Convergence, FTRA, vol. 5, no. 1, pp. 38-42, 2014.

[12] K. Peng, "A secure network for mobile wireless service," Journal of Information Processing Systems, vol. 9, no. 2, pp. 247-258, 2013. 

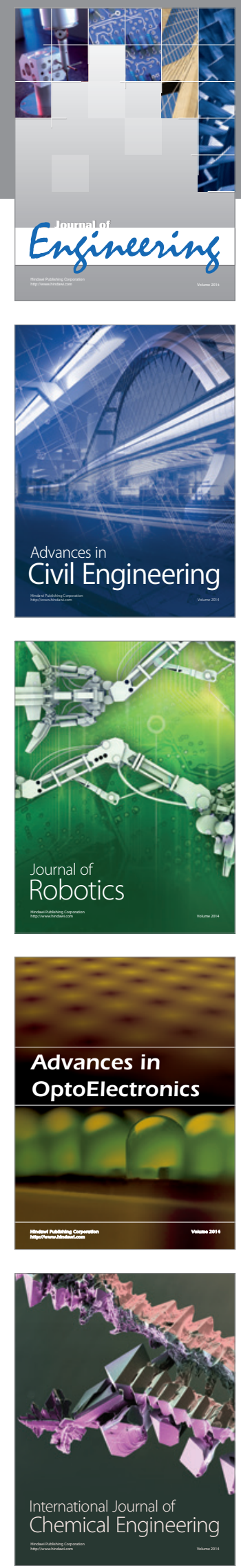

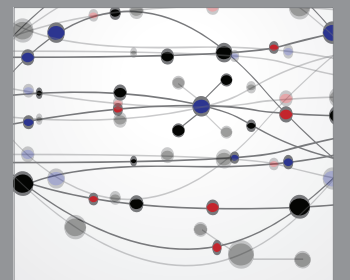

The Scientific World Journal
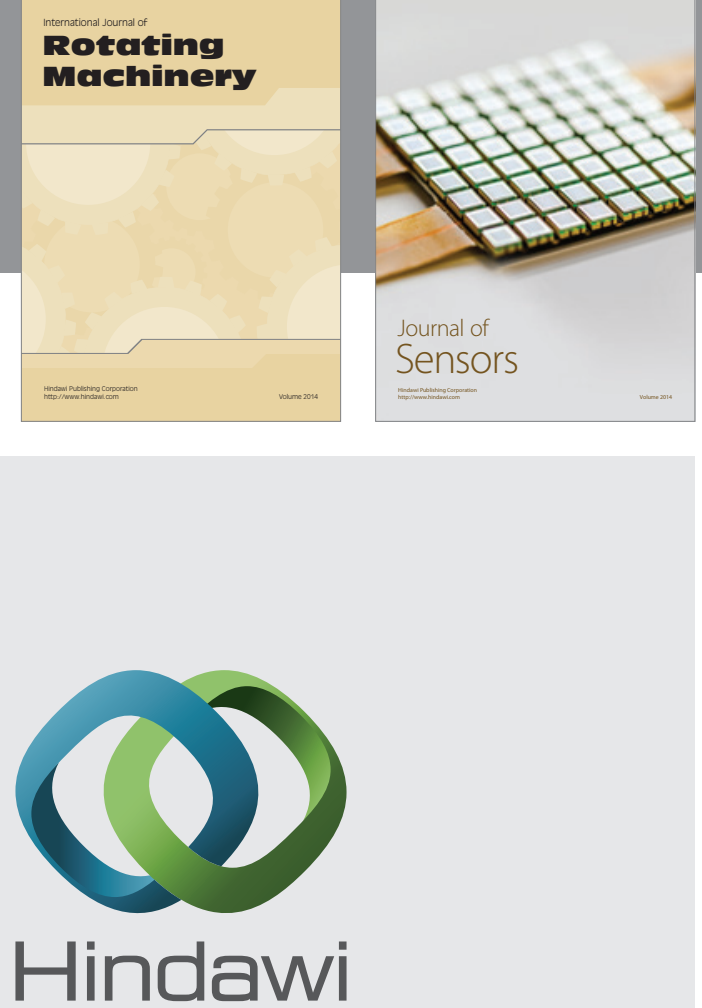

Submit your manuscripts at http://www.hindawi.com
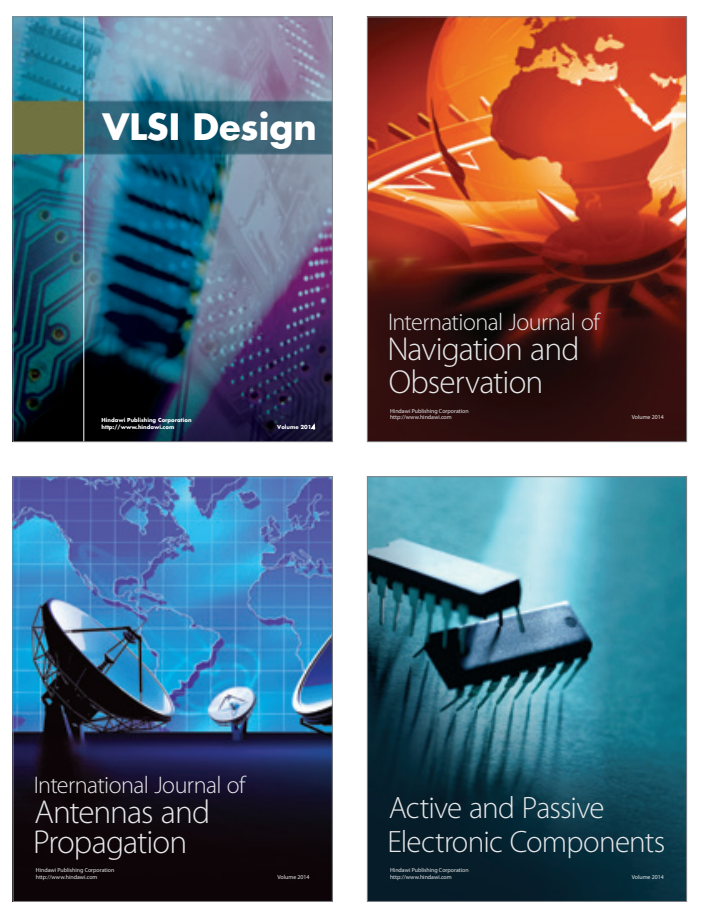
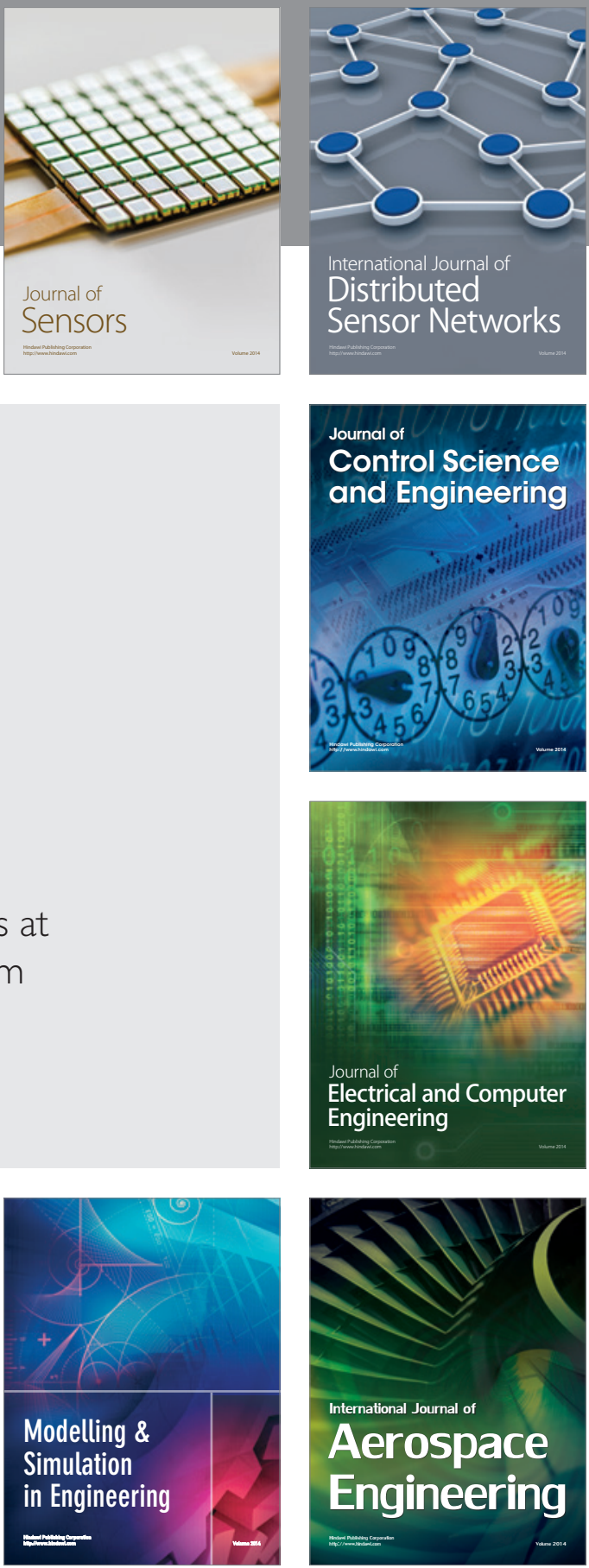

Journal of

Control Science

and Engineering
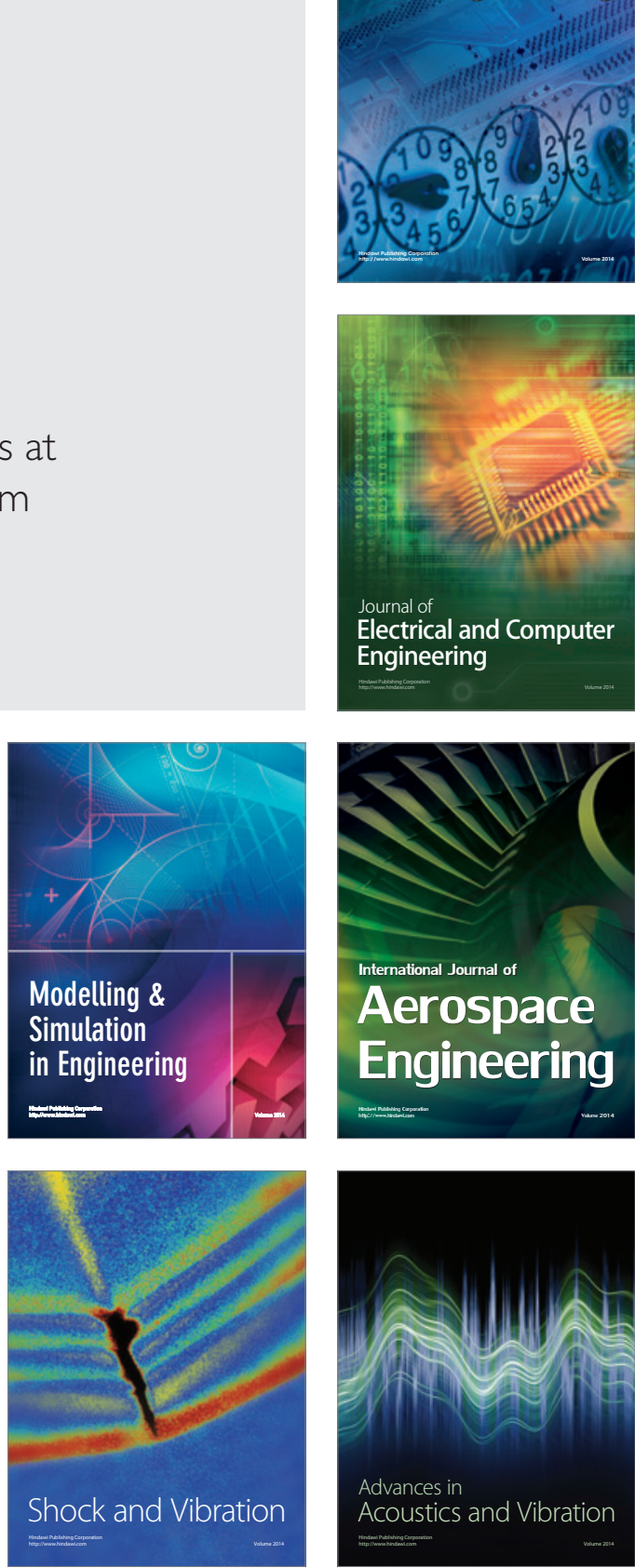